\title{
Reduced effective emotion regulation in night owls.
}

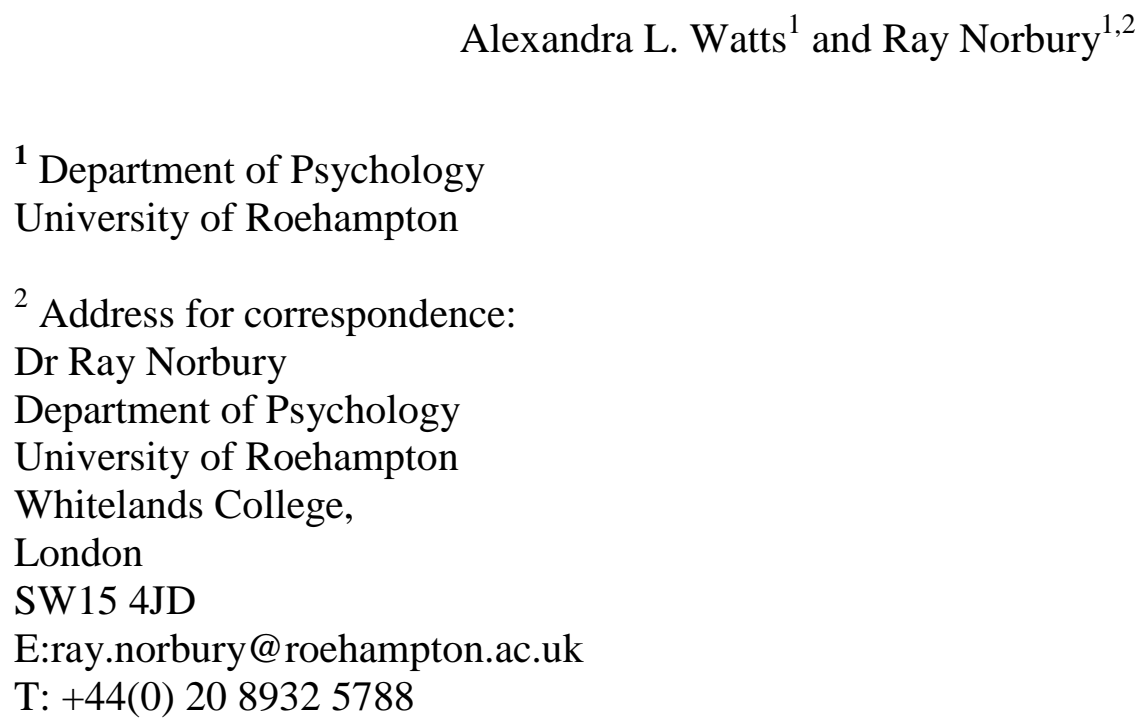




\begin{abstract}
Previous research has demonstrated a clear link between late chronotype and depression. The vulnerability factors underpinning this link, however, are unclear. Here the relationship between two specific emotion regulation strategies, cognitive reappraisal and expressive suppression, and chronotype was investigated using multiple regression. Two hundred and fourty participants (age range 18- 80, 189 females) completed validated selfreport questionnaires assaying chronotype, neuroticism, depression symptomatology, sleep quality and emotion regulation. Eveningness was associated increased expressive suppression and morningness was associated with increased cognitive reappraisal after controlling for age, gender, depressive symptomatology, neuroticism and sleep quality. Trait expressive suppression and reduced cognitive reappraisal are known to increase depression risk. Our results suggest that eveningness is associated with impaired emotion regulation which may confer risk for future depression. These findings suggest modifiable markers that could be therapeutically targeted to prevent the onset of depression in late chronotype individuals.
\end{abstract}

\title{
Introduction
}

Individual differences in sleep-wake behaviour (morningness-eveningness) and their relation to psychiatric disorder has attracted increasing attention in recent years (Fabbian et al., 2016). In particular, several studies have reported an association between eveningness and depression. Across the general population late chronotype is associated with increased depressive symptomatology (Levandovski et al., 2011; Merikanto et al., 2013, 2015; Togo, Yoshizaki, \& Komatsu, 2017), diagnosis of depression and use of antidepressant medication (Merikanto et al., 2013, 2015). Together, these data demonstrate a clear association between 
eveningness and depression but as they are observational they are insufficient to indicate the mechanisms whereby clinical depression emerges in late chronotype individuals.

A cardinal feature of depression is sustained negative affect. Indeed, to meet the Dianostic and Statistical Manual of Mental Disorders (DSM-5) criteria for a diagnosis of Major Depressive Disorder (MDD) requires the presence of either sustained negative affect or loss of pleasure for a two-week period. Individual differences in the habitual use of emotion regulation strategies may, therefore, play an important role in the onset and maintenance of depression and theorists argue that a failure to effectively manage emotional responses to everyday events leads to more prolonged and severe periods of distress which in turn evolve into clinical depression (Aldao, Nolen-Hoeksema, \& Schweizer, 2010)

Two specific emotion regulation strategies that have received significant attention are cognitive reappraisal and expressive suppression ( Gross \& John, 2003). Cognitive reappraisal, which involves the generation of a benign or more positive interpretation of a stressful event in order to reduce distress, is generally considered beneficial. Cognitive models of depression (Beck, Rush, Shaw, \& Emery, 1979) place maladaptive or ineffective reappraisal strategies at the core of depression and cognitivebehavioural therapies (CBT) for depression highlight adopting effective, adaptive reappraisal strategies. By contrast, expressive suppression, which involves inhibiting the emotional expression of the emotional experience, is generally considered maladaptive. Although effective at reducing the overt expression and possibly the subjective experience of emotion in the short-term, chronic use of this approach would be less effective at reducing emotional and physiological arousal (Gross \& John, 2003). More frequent use of expression suppression and inefficient reappraisal has been reported in non-clinical groups (Gotlib \& Joormann, 2010), currently depressed patients (Campbell-Sills, Barlow, Brown, \& Hofmann, 2006) and patients in remission (Aker, Harmer, \& Landrø, 2014; Ehring, Fischer, Schnülle, 
Bösterling, \& Tuschen-Caffier, 2008). More recently emotion dysregulation (as measured by the Difficulties in Emotion Regulation Scale [DERS]) has also been demonstrated in highly neurotic individuals (Paulus, Vanwoerden, Norton, \& Sharp, 2016). As high neuroticism is a widely recognised risk factor for depression (Vittengl, 2017) ineffective emotion regulation strategies may underlie, in part, the increased vulnerability of highly neurotic individuals for depression. However, to our best knowledge, no one has yet explored emotion regulation in relation to chronotype. Here we hypothesise that eveningness, which is associated with depression, will also be associated with reduced cognitive reappraisal and increased expressive suppression.

\section{Methods}

\section{Participants}

A total of 240 participants ( $M$ age $=25.97, \mathrm{SD}=11.74,181$ females $)$ completed an online survey (Qualtrics ${ }^{\circledR}$, Provo, UT). Of these, 150 were university students that received course credit for participation. The remaining participants were drawn from the general population and recruited via social media and personal communication. The survey was available between 1/11/2016 and 31/01/2017 and participants were free to complete the study at a time of their choosing with 75\% of surveys completed between 08:00 and 18:00. All procedures were approved by the local Research Ethics Committee (REC) and all participants provided informed consent prior to completing the survey.

\section{Questionnaires}

Chronotype was assessed using the 5-item reduced Morningness-Eveningness Questionnaire (rMEQ; Adan \& Almirall, 1991). The rMEQ consists of five items and participants are required to indicate their response on a 4-point scale (e.g “during the first half hour after you wake up in the morning, how do you feel?”:“very tired”, “fairly tired”, “fairly 
refreshed", and "very refreshed”). This scale has good internal consistency $(\alpha=.86)$ and higher scores indicate increased morningness. Sleep quality was determined using the Pittsburgh Sleep Quality Index (PSQI; Buysse, Reynolds, Monk, Berman, \& Kupfer, 1989). This scale produces a global sleep quality score, which is created by summing each of the subscales: subjective sleep quality, sleep latency, sleep duration, habitual sleep efficiency, sleep disturbance, use of sleeping medication, and daytime dysfunction. The PSQI has high internal consistency $(\alpha=.83)$. Items are answered either using a 4-point scale or by indicating time (e.g. “'During the last month, how often have you had trouble sleeping because you wake up in the middle of the night or early morning?”: "not during the last month”, "less than once a week”, “once or twice a week”, “more than three times a week”). Higher scores on the PSQI indicate worse sleep quality, with scores greater than 5 indicating clinically poor sleep quality. Neuroticism was assessed using the 12 neuroticism-related items taken from the revised Eysenck Personality Questionnaire (EPQ-R; Eysenck, Eysenck, \& Barrett, 1984). Participants are required to make a binary (yes/no response) to each item and neuroticism is calculated as the sum of yes responses. Emotion regulation was measured using the Emotion Regulation Questionnaire (ERQ; Gross \& John, 2003). The ERQ is a ten-item questionnaire designed to measure cognitive reappraisal and expression suppression (6 and 4 items each). Participants were instructed to indicate how they control their emotions by responding to each of the 10 items using a 7-point scale ranging from strongly agree to strongly disagree. The Cronbach alpha scores of the two subscales (reappraisal and suppression) are, respectively, .81 and .73). Depressive symptomatology was assessed using the Centre for Epidemiological Studies Depression Scale (CES-D; Radloff, 1977). Participants were required to indicate how often (rarely, some of the time, a moderate amount of time, all of the time) they have felt a certain way in the last 7 days (e.g. "During the past week I felt that everything I did was an effort”). The CES-D has high internal consistency $(\alpha=.85)$. 
Participants were also asked to indicate current or previous diagnosis of depression or sleep disorder.

\section{Statistical treatment}

Simple Pearson's correlations were used to examine associations between demographic characteristics, sleep quality, neuroticism, depressive symptomatology, current/previous depression, current/previous sleep disorder and chronotype. If significant at the bivariate level these predictors were included in a regression framework (Model 1). Model 2 subsequently examined the impact of emotion regulation (reappraisal and expressive suppression) on chronotype. All statistical analyses were carried out using the Statistcial Package for Social Sciences (SPSS) v21 (IBM, New York, USA).

\section{Results}

Basic demographics and sample characteristics are shown in Table 1. Cognitive

Table 1 near here please.

reappraisal and expressive suppression correlated with rMEQ at the bivariate level (respectively, $r(240)=.237, p<.001 ; r(240)=-.199, p=.002)$. Age, gender, depressive symptomatology, neuroticism and sleep quality were significantly correlated with rMEQ and

Table 2 near here please.

were thus entered as control variables in Model 1 . This model accounted for $18.4 \%$ of the variance in chronotype $(F(5,234)=10.56, p<.001)$. Increasing age was associated with morningness, whereas depressive symptomatology was associated with eveningness. Model 2 included age, gender, depressive symptomatology, neuroticism and sleep quality as well as cognitive reappraisal and expression suppression. This model explained $24.3 \%$ of the variance in chronotype and was a significantly better fit than Model $1(F(7,232)=10.66), p<$ 
.001). In this final model, morningness was associated with increased reappraisal and reduced expression suppression (please see Table 3).

Table 3 near here please.

Inspection of standard residuals suggested the data were not confounded by outliers (Std. Residual Min = -2.42, Std. Residual Max = 2.50). Assumptions of collineratity were met as tolearance values for all variables were greater than $0.1(0.452-0.947)$ and VIF scores were less than $10(1.06-2.21)$. The data met the assumption of independent errors (DurbinWatson value $=1.99)$ and a histogram of standardised residuals indicated that the data contained approximately normally distributed errors as did the normal P-P plot of standardised residuals. A scatterplot of standardised predicted values showed that the data met the assumptions of homogeneity of variance and linearity. Finally, the data also met the assumption of non-zero variances $(13.18-137.23)$

\section{Discussion}

A burgeoning corpus suggests that late chronotype is associated with depression, antidepressant use and depressive symptoms. Disordered affect (characterised by sustained negative affect and reduced experience of positive affect) is a cardinal feature of depression and individual differences in emotion regulation have been linked to increased risk for depression. Here we show that eveningness was associated with maladaptive emotion regulation strategies (reduced cognitive reappraisal and increased expression suppression) after controlling for age, gender, depressive symptomatology, neuroticism and sleep quality. Inefficient emotion regulation may, therefore, underlie in part the increased vulnerability of late chronotypes for psychiatric disorder. 
Recent important population studies provide good evidence for a link between late chronotype and depression (Merikanto et al., 2013, 2015). However, the observational nature of those studies precludes determining the underlying psychological mechanisms that underpin this link. The vulnerability-stress hypothesis of depression posits that depression emerges through an interaction between a psychological-vulnerability (e.g. preferential processing of negative material) and an environmental stressor (e.g. death of a loved one, job loss) and one approach to explore this hypothesis is to investigate cognitive biases in 'at-risk' groups. Using this method Chan and colleagues (Chan, Goodwin, \& Harmer, 2007) reported that high neuroticism (a recognised risk factor for depression) was associated with reduced latency to classify negative versus positive personality descriptors, reduced positive memory intrusion at subsequent recall and reduced capacity to recognise happy facial expressions as compared to individuals with low levels of neuroticism. Using the same 'at-risk' model with late chronotypes Berdynaj and colleagues reported increased recognition of sad facial expressions, greater recall and reduced latency to correctly recognise previously presented negative personality trait words and reduced allocation of attentional resources to happy faces in late chronotypes (Berdynaj et al., 2016). Similarly, Horne et al., (Horne, Marr-Phillips, Jawaid, Gibson, \& Norbury, 2016) reported increased recognition accuracy in late chronotypes for sad compared to happy faces independent of sleep quality, mood, age and gender. Emerging evidence (Berdynaj et al., 2016; Horne et al., 2016), therefore, suggests that in healthy, never-depressed individuals, late chronotype is associated with negative biases in emotional processing which if combined with adversity may lead to depression.

In the current study we also found that morningness was associated with adaptive antecedent-focused emotion regulation strategies (cognitive reappraisal) where action is taken prior to the emotion response tendency becoming fully activated. By contrast, eveningness was associated with expressive suppression (generally considered maladaptive), a response- 
focused strategy that is instigated once an emotion is underway. The consequences related to this pattern of emotion regulation are likely far reaching. Expressive suppression is considered cognitively more demanding as this approach requires constant updating to manage and maintain emotional responses thereby monopolising valuable cognitive resources that would be better spent on other tasks (John \& Gross, 2004). In addition, the conflict generated between the overt 'controlled' behaviour and inner emotion and the sense of not being true to oneself may itself lead to increased negativity thereby contributing to continuing depressed mood (Sheldon, Ryan, Rawsthorne, \& Ilardi, 1997).

To test the affective consequences of reappraisal and suppression Gross (1998) invited participants to view movie clips designed to elicit negative emotions. Participants in the control group simply watched the film (no instruction), in the reappraisal condition participants were instructed to think about the film in such a way that they would feel nothing and in the suppression condition participants were instructed to behave in such a way that an independent viewer would not know they were feeling anything. Compared to the control condition, both suppression and reappraisal were effective in reducing emotion-expressive behaviour. Suppression, however, had no effect on the subjective experience of negative emotion. That is, although outwardly participants in the suppression group appeared unmoved they felt as much emotion as the no instruction, control group. In addition, physiologically, suppression was associated with increased sympathetic activation (increased heart rate and galvanic skin response). By contrast, reappraisal reduced both the experience and expression of negative emotion and had no impact on physiological arousal. These data and results from related studies (Gross \& Levenson, 1993; Gross \& Levenson, 1997) suggest that expressive suppression, here associated with eveningness, does not lead to a reduction in the experience of emotion and may feed into a spiral of despair and negativity. Cognitive 
reappraisal however (here associated with morningness) has a positive impact and is effective at reducing the subjective experience of negative emotion.

The current findings and previous work suggest that eveningness is associated with impaired emotional processing and regulation (Berdynaj et al., 2016; Horne et al., 2016) and suggests possible interventions for the prevention of depression in late chronotype individuals. However, the underlying causes that lead to depression are likely multifactorial and there is a need for longitudinal studies to explore and determine effective strategies that promote psychological well-being in these individuals. For example, experimental manipulations that allow evening-types to follow, or better match, their circadian rhythm may be effective in reducing depressive symptomatology. Alternatively, correcting phase disturbance with bright morning light, melatonin or melatonin agonists may also be useful strategies to improve mood. Indeed, agomelatine, an agonist at melatonergic $\left(\mathrm{MT}_{1} / \mathrm{MT}_{2}\right)$ receptors, has been demonstrated to be an effective antidepressant (Kasper et al., 2010). In addition to our novel findings of reduced cognitive reappraisal and increased expressive suppression in evening type individuals our data are in good agreement with previous research exploring chronotype. For example, we observed significant correlations between age, gender, depressive symptomatology, neuroticism and sleep quality. Morning types tended to be older, female, report lower depressive symptomatology and neurotic traits and have better sleep quality - data that are consistent with previous work in this field (Duggan, Friedman, McDevitt, \& Mednick, 2014; Merikanto et al., 2013, 2015; Randler, Freyth-Weber, Rahafar, Florez Jurado, \& Kriegs, 2016).

There are a number of limitations associated with this study which should be taken into account when considering the findings. First, chronotype status was determined using a single metric, the Reduced Morningness-Eveningness Questionnaire (Adan \& Almirall, 1991), future studies may benefit from objective tools such as acrophase estimates of cortisol 
and core body temperature, polysomnography and actigraphy to corroborate self-report questionnaire data. Also, the ability of individuals to accurately self-report emotion regulation strategies has been scrutinised (Robinson \& Clore, 2002). Respondents may be influenced by negative mood or other biases, they may also confound the experience of emotion with its regulation and the degree of insight and meta-cognition required to complete the questionnaires may be challenging for some participants. Future work that includes experimental measures to assess the affective consequences of different emotion regulation strategies (e.g. viewing video clips designed to elicit negative emotion (John \& Gross, 2004) supported by self-report questionnaires are warranted. In addition, our anonymous online survey required that current and/or previous depression and diagnosed sleep disorder were determined by self-report. Future studies that include reference to medical history, structured clinical interview and other known risk factors for depression such family history of depression and stressful life events are required. Also, our study sample was predominantly female (78\%) and it has been reported that emotion regulation strategies vary as a function on menstural cyle with the mid-late luteal phase and follicular phase being associated with reduced cognitive reappraisal as compared to the menstrual phase (Wu, Zhou, \& Huang, 2014). Here, we did not record menstrual cycle phase nor did we conduct repeat testing (e.g. to compare emotion regulation during the follicular and luteal phase). We cannot, therefore, exclude a potential impact of cycle phase on our findings. Finally, a limited number of studies have explored the potential link between eveningness, depression and body mass index [BMI]. In adolescent females Pabst and colleagues (2009) reported that eveningness was associated with greater depressive symptomatology, as measured by the Children's Depression Inventory, in both normal and overweight girls although the effect was more marked in overweight individuals (Pabst, Negriff, Dorn, Susman, \& Huang, 2009). More recently, data from a large adult population study Merikanto et al., (2015) suggest that 
eveningness was associated with reduced BMI. Moreover, stratification of morning, intermediate and evening types by number of depressive symptoms revealed that, compared to morning types, evening type individuals with none or 1 depressive symptom had lower BMI scores whereas BMI did not distinguish between chronotype groups when greater levels of depression (two symptoms present) were present (Merikanto et al., 2015). Taken together (Merikanto et al., 2015; Pabst et al., 2009) these data suggest that the relationship between circadian preference, depressive symptomatology and BMI is complex and varies as a function of age. In the current study we did not measure BMI. However, the observed association between eveningness and reduced cognitive reappraisal/increased expressive suppression remained significant after controlling for depressive symptoms. Future studies may benefit from including measures of BMI, especially if the target population includes adolescents or young adults.

In conclusion, the current findings demonstrate impaired emotion regulation in late chronotypes that may confer risk for future depression. Specifically, we found that morningness was associated with cognitive reappraisal, widely theorised to be protective against psychopathology, and eveningness was associated with expressive suppression which has been argued to be a risk factor for psychopathology. These findings suggest modifiable markers that could be therapeutically targeted to prevent the onset of depression in late chronotype individuals. 


\section{References}

Adan, A., \& Almirall, H. (1991). Horne \& Östberg morningness-eveningness questionnaire: A reduced scale. Personality and Individual Differences, 12(3), 241-253. https://doi.org/10.1016/0191-8869(91)90110-W

Aker, M., Harmer, C., \& Landrø, N. I. (2014). More rumination and less effective emotion regulation in previously depressed women with preserved executive functions. BMC Psychiatry, 14(1), 1.

Aldao, A., Nolen-Hoeksema, S., \& Schweizer, S. (2010). Emotion-regulation strategies across psychopathology: A meta-analytic review. Clinical Psychology Review, 30(2), 217-237. https://doi.org/10.1016/j.cpr.2009.11.004

Beck, A. T., Rush, A. J., Shaw, B. F., \& Emery, G. (1979). Cognitive theory of depression. New York: Guildford.

Berdynaj, D., Boudissa, S. N., Grieg, M. S., Hope, C., Mahamed, S. H., \& Norbury, R. (2016). Effect of chronotype on emotional processing and risk taking. Chronobiology International, 1-13. https://doi.org/10.3109/07420528.2016.1146739

Buysse, D. J., Reynolds, C. F., 3rd, Monk, T. H., Berman, S. R., \& Kupfer, D. J. (1989). The Pittsburgh Sleep Quality Index: a new instrument for psychiatric practice and research. Psychiatry Research, 28(2), 193-213.

Campbell-Sills, L., Barlow, D. H., Brown, T. A., \& Hofmann, S. G. (2006). Acceptability and suppression of negative emotion in anxiety and mood disorders. Emotion (Washington, D.C.), 6(4), 587-595. https://doi.org/10.1037/1528-3542.6.4.587

Chan, S., Goodwin, G. M., \& Harmer, C. J. (2007). Highly neurotic never-depressed students have negative biases in information processing. Psychological Medicine, 37(9), 12811291. https://doi.org/10.1017/S0033291707000669 
Duggan, K. A., Friedman, H. S., McDevitt, E. A., \& Mednick, S. C. (2014). Personality and Healthy Sleep: The Importance of Conscientiousness and Neuroticism. PLoS ONE, 9(3), e90628. https://doi.org/10.1371/journal.pone.0090628

Ehring, T., Fischer, S., Schnülle, J., Bösterling, A., \& Tuschen-Caffier, B. (2008). Characteristics of emotion regulation in recovered depressed versus never depressed individuals. Personality and Individual Differences, 44(7), 1574-1584. https://doi.org/10.1016/j.paid.2008.01.013

Eysenck, S. B. ., Eysenck, H. ., \& Barrett, P. (1984). A revised version of the psychotism scale. Personality and Individual Differences, 6(1), 21-29.

Fabbian, F., Zucchi, B., Giorgi, A. D., Tiseo, R., Boari, B., Salmi, R., ... Manfredini, R. (2016). Chronotype, gender and general health. Chronobiology International, 1-20. https://doi.org/10.1080/07420528.2016.1176927

Gotlib, I. H., \& Joormann, J. (2010, March 24). Cognition and Depression: Current Status and Future Directions [review-article]. Retrieved 29 January 2017, from http://www.annualreviews.org/doi/10.1146/annurev.clinpsy.121208.131305

Gross, J. J., \& John, O. P. (2003a). Individual differences in two emotion regulation processes: Implications for affect, relationships, and well-being. Journal of Personality and Social Psychology, 85(2), 348-362. https://doi.org/10.1037/00223514.85.2.348

Gross, J. J., \& John, O. P. (2003b). Individual differences in two emotion regulation processes: Implications for affect, relationships, and well-being. Journal of Personality and Social Psychology, 85(2), 348-362. https://doi.org/10.1037/00223514.85.2.348

Gross, J. J., \& Levenson, R. W. (1993). Emotional suppression: physiology, self-report, and expressive behavior. Journal of Personality and Social Psychology, 64(6), 970-986. 
Gross, J., \& Levenson, R. W. (1997). Hiding feelings: the acute effects of inhibiting negative and positive emotion. Journal of Abnormal Psychology, 106(1), 95.

Horne, C., Marr-Phillips, S. D. M., Jawaid, R., Gibson, E. L., \& Norbury, R. (2016). Negative emotional biases in late chronotypes. Biological Rhythm Research, 1-5. https://doi.org/10.1080/09291016.2016.1236461

John, O. P., \& Gross, J. J. (2004). Healthy and unhealthy emotion regulation: personality processes, individual differences, and life span development. Journal of Personality, 72(6), 1301-1333. https://doi.org/10.1111/j.1467-6494.2004.00298.x

Kasper, S., Hajak, G., Wulff, K., Hoogendijk, W. J. G., Montejo, A. L., Smeraldi, E., ... Baylé, F. J. (2010). Efficacy of the novel antidepressant agomelatine on the circadian rest-activity cycle and depressive and anxiety symptoms in patients with major depressive disorder: a randomized, double-blind comparison with sertraline. The Journal of Clinical Psychiatry, 71(2), 109-120. https://doi.org/10.4088/JCP.09m05347blu

Levandovski, R., Dantas, G., Fernandes, L. C., Caumo, W., Torres, I., Roenneberg, T., ... Allebrandt, K. V. (2011). Depression Scores Associate With Chronotype and Social Jetlag in a Rural Population. Chronobiology International, 28(9), 771-778. https://doi.org/10.3109/07420528.2011.602445

Merikanto, I., Kronholm, E., Peltonen, M., Laatikainen, T., Vartiainen, E., \& Partonen, T. (2015). Circadian preference links to depression in general adult population. Journal of Affective Disorders, 188, 143-148. https://doi.org/10.1016/j.jad.2015.08.061

Merikanto, I., Lahti, T., Kronholm, E., Peltonen, M., Laatikainen, T., Vartiainen, E., ... Partonen, T. (2013). Evening types are prone to depression. Chronobiology International, 30(5), 719-725. https://doi.org/10.3109/07420528.2013.784770 
Pabst, S. R., Negriff, S., Dorn, L. D., Susman, E. J., \& Huang, B. (2009). Depression and Anxiety in Adolescent Females: The Impact of Sleep Preference and Body Mass Index. Journal of Adolescent Health, 44(6), 554-560. https://doi.org/10.1016/j.jadohealth.2008.11.012

Paulus, D. J., Vanwoerden, S., Norton, P. J., \& Sharp, C. (2016). Emotion dysregulation, psychological inflexibility, and shame as explanatory factors between neuroticism and depression. Journal of Affective Disorders, 190, 376-385. https://doi.org/10.1016/j.jad.2015.10.014

Radloff, L. S. (1977). The CES-D Scale A Self-Report Depression Scale for Research in the General Population. Applied Psychological Measurement, 1(3), 385-401. https://doi.org/10.1177/014662167700100306

Randler, C., Freyth-Weber, K., Rahafar, A., Florez Jurado, A., \& Kriegs, J. O. (2016). Morningness-eveningness in a large sample of German adolescents and adults. Heliyon, 2(11), e00200. https://doi.org/10.1016/j.heliyon.2016.e00200

Robinson, M. D., \& Clore, G. L. (2002). Episodic and semantic knowledge in emotional selfreport: evidence for two judgment processes. Journal of Personality and Social Psychology, 83(1), 198-215.

Sheldon, K. M., Ryan, R. M., Rawsthorne, L. J., \& Ilardi, B. (1997). Trait self and true self: Cross-role variation in the Big-Five personality traits and its relations with psychological authenticity and subjective well-being. Journal of Personality and Social Psychology, 73(6), 1380. https://doi.org/10.1037/0022-3514.73.6.1380

Togo, F., Yoshizaki, T., \& Komatsu, T. (2017). Association between depressive symptoms and morningness-eveningness, sleep duration and rotating shift work in Japanese nurses. Chronobiology International, 1-11. https://doi.org/10.1080/07420528.2016.1273942 
Vittengl, J. R. (2017). Who pays the price for high neuroticism? Moderators of longitudinal risks for depression and anxiety. Psychological Medicine, 1-12.

https://doi.org/10.1017/S0033291717000253

Wu, M., Zhou, R., \& Huang, Y. (2014). Effects of menstrual cycle and neuroticism on females’ emotion regulation. International Journal of Psychophysiology: Official Journal of the International Organization of Psychophysiology, 94(3), 351-357. https://doi.org/10.1016/j.ijpsycho.2014.10.003 